\title{
Experimental Optimization of the Output Power of a Copper Vapor Laser Using Air as a Buffer Gas
}

\author{
Mahboobeh Mirzaei', Saeid Behrouzinia ${ }^{2 *}$, Masoud Sabaghi², Saeid Marjani' ${ }^{3}$, \\ Kamran Khorasani' ${ }^{2}$, Batool Sajad ${ }^{1}$ \\ ${ }^{1}$ Department of Physics, Alzahra University, Tehran, Iran \\ ${ }^{2}$ Laser and Optics Research School, Nuclear Science and Technology Research School, Atomic Energy \\ Organization of Iran, Tehran, Iran \\ ${ }^{3}$ Department of Electrical Engineering, Ferdowsi University of Mashhad, Mashhad, Iran \\ Email: "sbehrouzi@aeoi.org.ir
}

Received 4 March 2016; accepted 18 April 2016; published 21 April 2016

Copyright (C) 2016 by authors and Scientific Research Publishing Inc.

This work is licensed under the Creative Commons Attribution International License (CC BY).

http://creativecommons.org/licenses/by/4.0/

c) (i) Open Access

\begin{abstract}
In order to investigate the effect of the pressure buffer gas and frequency on the output power, a copper vapor laser with active medium length of $60 \mathrm{~cm}$ and bore of $16 \mathrm{~mm}$ has been operated and optimized using air as a buffer gas. The observed oscillatory behavior of the output power versus frequency is in good agreement with the previous reports. The measured results show the maximum output power of $\sim 1.6 \mathrm{~W}$ at the optimum pressure of $3.8 \mathrm{torr}$ and frequency of $17 \mathrm{kHz}$. Abundance of the air and reduction of the system volume due to elimination of the gas handling system as well as the economically benefits are the advantages of the employing air as a buffer gas in the copper vapor laser operation.
\end{abstract}

\section{Keywords}

Experimental Optimization, Copper Vapor Laser, Air Buffer Gas

\section{Introduction}

The lasers are devices that emit light coherently through a process of optical amplification based on the stimulated emission of photons. There are many different kinds of lasers for different and specific uses [1]-[28]. One of the lasers with high average power in the visible region at $510.6 \mathrm{~nm}$ and $578.2 \mathrm{~nm}$ is the copper vapor laser

\footnotetext{
${ }^{*}$ Corresponding author.
}

How to cite this paper: Mirzaei, M., Behrouzinia, S., Sabaghi, M., Marjani, S., Khorasani, K. and Sajad, B. (2016) Experimental Optimization of the Output Power of a Copper Vapor Laser Using Air as a Buffer Gas. Optics and Photonics Journal, 6, 5359. http://dx.doi.org/10.4236/opj.2016.64008 
(CVL). Applications of CVL include the research, medicine, industry, high speed imaging, micro-machining, non-linear frequency conversion to the ultraviolet and photodynamic therapy. Furthermore, they have been used extensively as a powerful pumping source for dye lasers by the master oscillator-power amplifier array for higher output powers and beam quality [29]-[34]. In order to increase the output power and improve the laser performance, the buffer gas is used in CVL tube that provides a discharge medium when the discharge tube reaches to the required temperature of copper vapor production. The buffer gas provides the population inversion mechanism by means of energy transfer of the gas, ions or electrons to the metal atoms. On one hand, it shapes the energy distribution function through elastic collisions and on the other hand, it protects the windows against contamination and then transfers heat from the tube axis to the walls. Therefore, the buffer gas causes the increase in impedance of the discharge tube and consequently leads to improving matching by the excitation circuit [30]. In order to minimize the effect of inelastic electron collisions on the energy distribution function, the non-reactive inert gases with high excitation and ionization energies have been employed as the buffer gases such as He, Ne, Ar, Kr, Xe and their mixtures gases. He gives longer and lower discharge current pulses [30]. Neon enables laser oscillation at the highest gas pressure and obtained the highest output power among of them [35]. The weak oscillations are provides with $\mathrm{N}_{2}$ [36], $\mathrm{H}_{2}$, and $\mathrm{CO}_{2}$ buffers [37]. The Ne buffer gas with adding of $\mathrm{H}_{2}$ extremely improves CVL performance [38] [39]. Recently, we reported the gold vapor laser with Heas a buffer gas to comparison output power and the stability [40]. In this work, a CVL has been operated using air as a buffer gas and the behavior of the laser output power versus discharge frequency and gas pressure have been investigated to obtain the optimum conditions. Furthermore, formed layer and pollutions on the tube laser has been analyzed by XRD instrument and FTIR spectrometer to find layer compositions. Affluence of the air and reduction of the system volume due to eliminating of the gas handling as well as the thriftily benefits are the advantages of the using air as a buffer gas in the CVL operation in some researchers or educational cases. The paper is organized as follows: the experimental setup is described in Section 2. Section 3 presents the obtained results and discussion. The concluding remarks are given in Section 4.

\section{Experimental Setup}

The material of discharge tube is Alumina that its inner diameter and active medium length are $16 \mathrm{~mm}$ and 60 $\mathrm{cm}$, respectively. It surrounded by a fibrous alumina insulation covering with a Pyrex tube and an external stainless steel jacket. In order to discharging, two molybdenum cylindrical electrodes are used. The back and front mirrors are consist of the couple of flat-flat resonator with reflectivity of $98 \%$ and $4 \%$, respectively. As illustrated in Figure 1, the standard driven circuit is coupled to the laser tube. By the discharge of a 1.65-nF main capacitor $\left(C_{s}\right)$ through the TGI1-1000/25 Thyratron, the tube gas is excited. A 0.68-nF peaking capacitor $\left(C_{p}\right)$ is connected between the tube electrodes as well as the $0.15 \mathrm{mH}$ inductance $\left(L_{2}\right)$.

The experimental characteristics of the laser output are measured by a Tektronix ${ }^{\mathrm{TM}}$ oscilloscope; model TDS1250, a Molectron ${ }^{\mathrm{TM}}$ PM500D powermeter, and a semiconductor detector model pbx-65. A FTIR spectrometer and XRD instrument are exploited to determine the chemical elements level to study the composition of the formed layer at the ends of laser tube.

\section{Results and Discussion}

The CVL has been operated by employing the air as a buffer gas to investigate and optimized the output power in terms of the various parameters at the constant input power of $\sim 1.4 \mathrm{~kW}$. At first, the behavior of the output power versus frequency has been studied at constant air pressure. The experiments are repeated at some various air pressures. The obtained results at some typical pressures of 2, 3.8, 5.3, 6.2 and 7.8 torr are shown in Figure 2. There is an optimum pressure and frequency for each CVL, which gives maximum output power. The optimum pressure is due to rate of collision between air molecules and copper atoms, which at optimum pressure, the population inversion and so gain of Laser would be increased, as for the other conventional buffer gases has been occurred. The optimum frequency and oscillatory behavior comes from geometrical structure of tube only, and independent of kind of buffer gas. The maximum output power of $\sim 1.6 \mathrm{~W}$ is obtained at 3.8 torr of air pressure and $17 \mathrm{kHz}$ of frequency. The noticeable point is the oscillatory behavior of the output power in terms of the frequency, which is in agreement with the previous reports [41] [42]. The effect arises from the laser tube which performs both as an optical and an acoustic resonator at some frequencies. In fact, the discharge energy extracted by optical and acoustical channels due to radiative and non-radiative decays, respectively. It is shown 


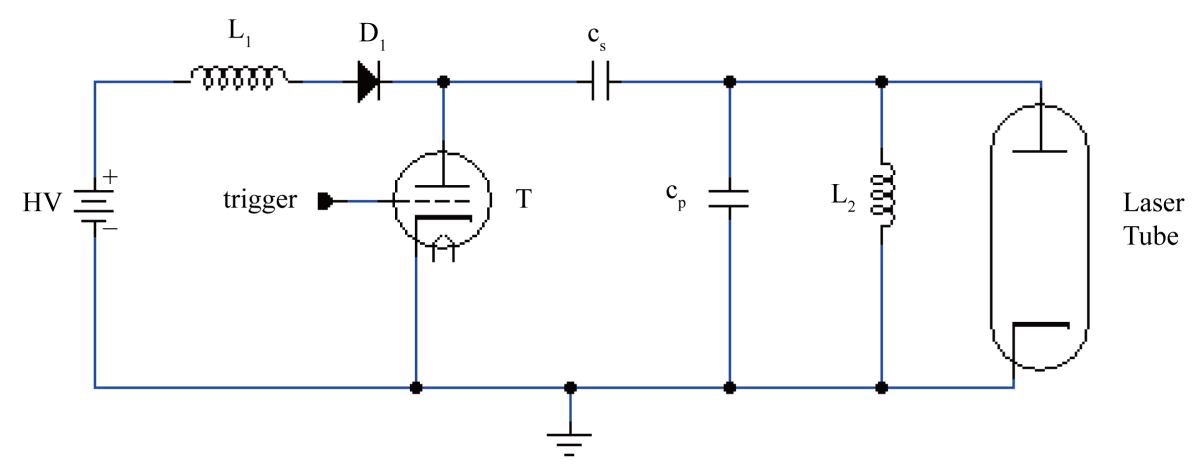

Figure 1. Schematic layout of standard driven circuit of CVL.

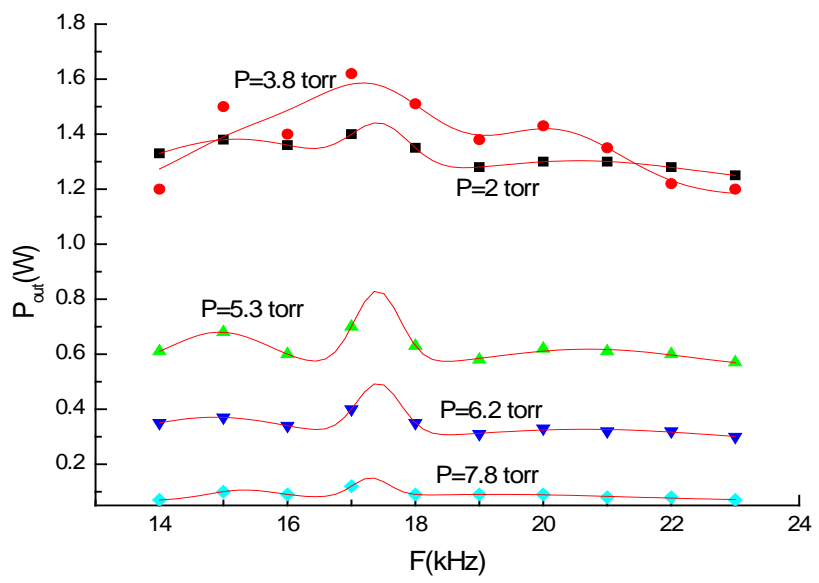

Figure 2. The output power versus frequency at some various air pressures.

that the non-radiative deactivation vastly increases, when the excitation frequency is equivalent to the acoustic resonant mode of laser tube [41].

Figure 3 illustrates the behavior of the output power versus the pressure of air buffer gas at some frequencies. At first, the output power increases by increasing the air pressure for all frequencies. After reaching to a maximum value, it reduces at higher pressures. The reduction of the output power is due to increases in collision and relaxation of excited copper atoms. It is seen that the maximum output power at all frequencies occurs at $\sim 3.8$ torr of the air pressure. Compared with other buffer gases like He and Ne, the output power of CVL with air as buffer gas is decreased in the same discharge frequency due to exuberance of impurities in air, which alter the condition of population inversion. Meanwhile the optimum Ne pressure for this laser is about 25 torr that is 6 times more than that of air one. Furthermore, it is observed that the peak power is reduced from 1.6 to $1.27 \mathrm{~W}$ by increasing of frequency from 17 to $22 \mathrm{kHz}$ due to the increasing in the inter pulse population of the lower laser level and the inter pulse electron density.

Abundance of the air and reduction in volume of the laser system due to elimination of the gas handling as well as the economic benefits are the advantages of the air where is using as a buffer gas in the CVL operation, in some research or educational specific cases. However, the laser tube is polluted after some operation such that some populace in red color are deposited at one of the tube end and a black color one is deposited at the other side, as shown in Figure 4. The Laser tube is polluted after some operation, which causes the lifetime of the tube would decrease the output power of CVL, and no effect on frequency operation. However, pollution can be eliminated by cleaning of tube through removal of pollutant.

The deposited samples at each ends of the laser tube are analyzed by FTIR spectrum and XRD instruments to determine the component of composition. Figure 5 displays FTIR spectrum before and after some laser operation.

Based on the FTIR spectrum, the component of composition in formed layer at the ends of the tube can be 


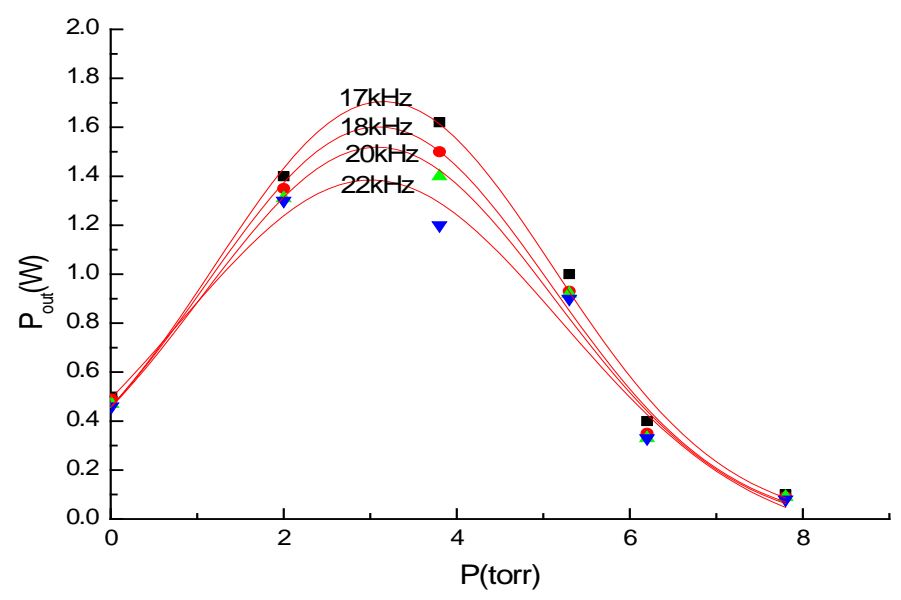

Figure 3. The output power versus air buffer gas pressures at some frequencies.

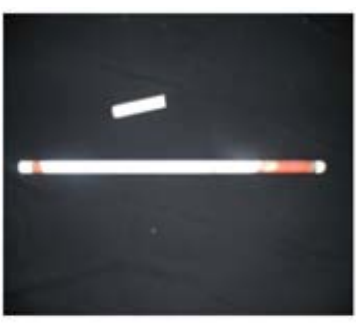

(a)

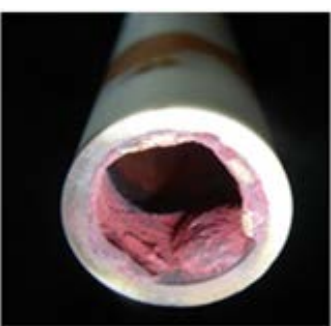

(b)

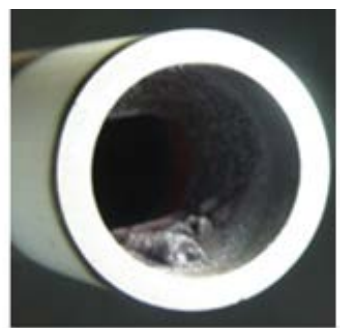

(c)

Figure 4. The laser tube after some operation of CVL using air as a buffer gas.

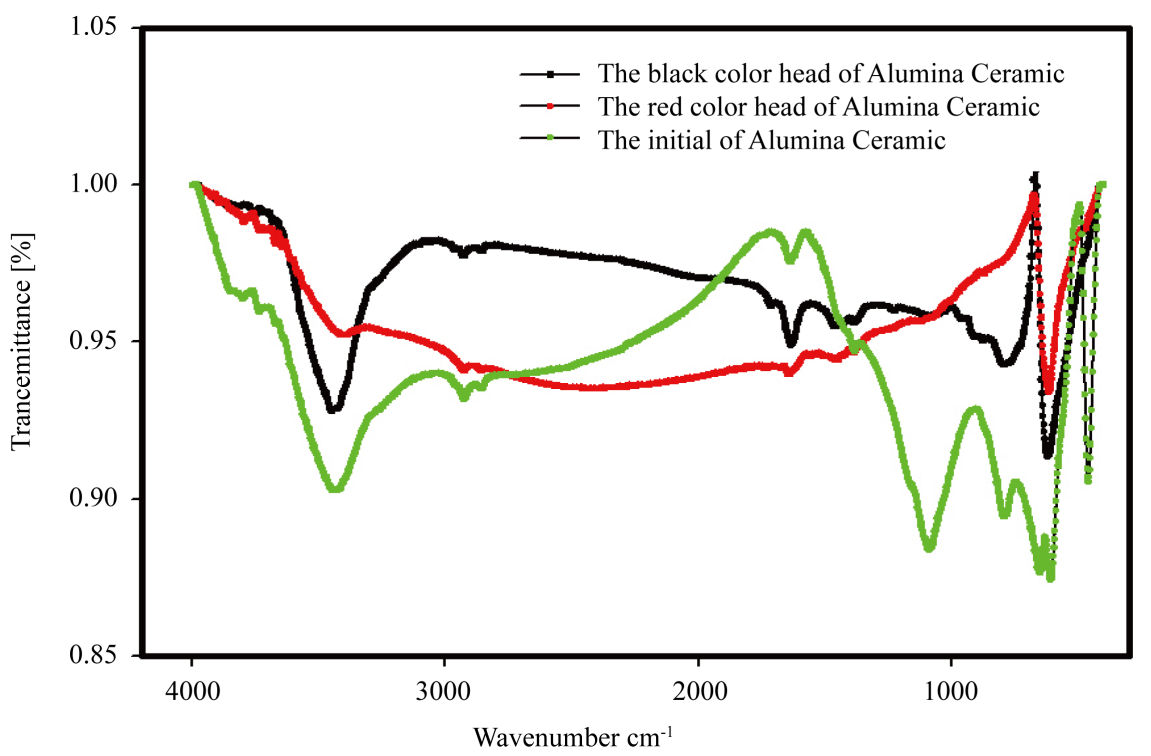

Figure 5. FTIR spectrum of the laser tube.

determined to be copper oxide in the red side and the copper oxide, the copper aluminum oxide and the copper sulfate hydroxide at the other one. It is emphasized by XRD spectrums, as shown in Figure 6.

\section{Conclusion}

In this work, a CVL laser was employed using air as a buffer gas. The behavior of the output power is investigated 


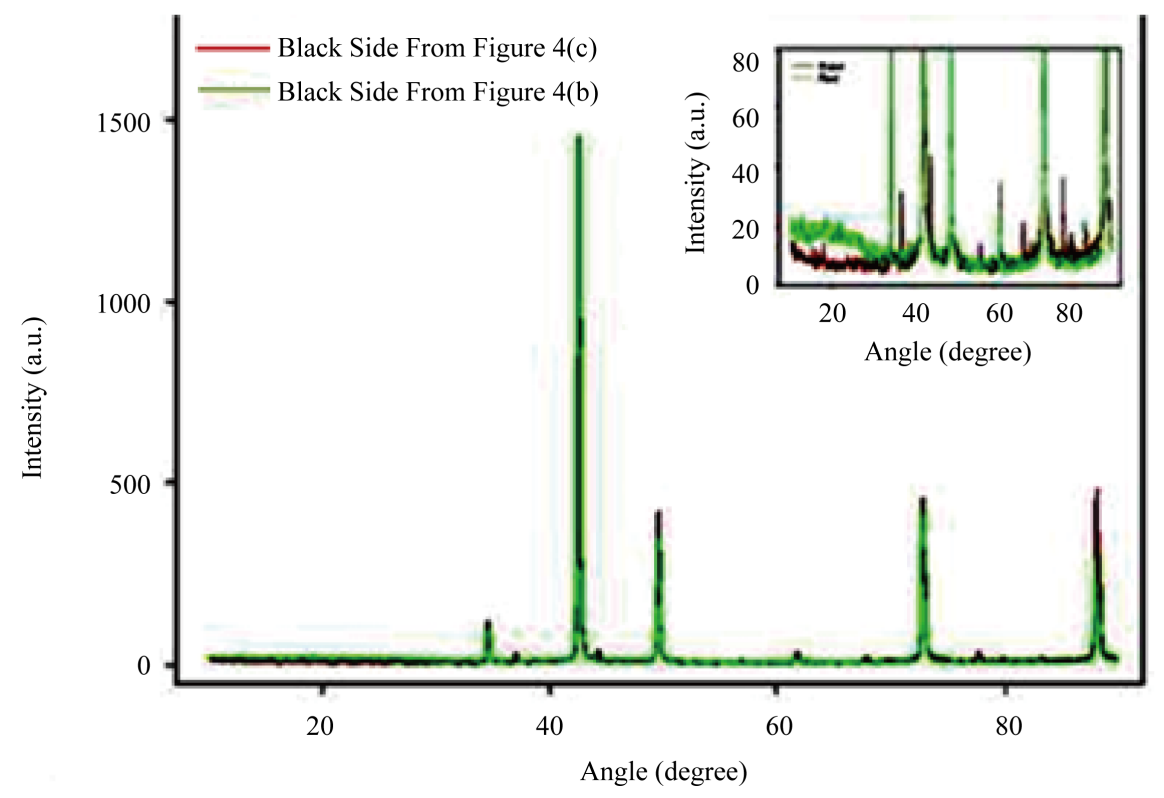

Figure 6. XRD spectrum of the laser tube. Black and redsides indicate copper oxide; and copper oxide and copper aluminum oxide and copper sulfate hydroxide, respectively.

in terms of the discharge frequency and gas pressure to determine the optimum condition. The oscillatory behavior of the output power versus frequency is observed which is in agreement with previous reports. The output power versus pressure is also studied to measure the maximum output power of $\sim 1.6 \mathrm{~W}$ at the optimum pressure of 3.8 torr and at $17 \mathrm{kHz}$ of frequency. Abundance and easy access of air as buffer gas and reduction of the weight volume of the laser system due to eliminating the gas handling as well as the economic benefits are the advantages of the air as a buffer gas for some research or educational specific cases. However, by using air as a buffer gas, the lifetime of the tube would be decreased.

\section{References}

[1] Marjani, S. (2013) Various Elements of Heat Sources within an Optimized Photonic Crystal Vertical Cavity Surface Emitting Laser: Influence of Hole Etching Depth. Asian Journal of Chemistry, 25, 4153-4156. http://dx.doi.org/10.14233/ajchem.2013.13281

[2] Rafighi, F., Behrouzinia, S., Khorasani, K., Sabaghi, M. and Marjani, S. (2016) The Electrical Parameters Modeling and Experimentation of Copper Vapor Laser. Circuits and Systems, 7, 23-28. http://dx.doi.org/10.4236/cs.2016.71003

[3] Behrouzinia, S., Khorasani, K., Marjani, S., Sabaghi, M., Aeinehvand, M.E. and Mohammadpour Lima, S. (2016) Experimental Study of Buffer Gas Flow Rate Effect on Output Power of a Copper Vapor Laser. Optics and Photonics Journal, 6, 24-28. http://dx.doi.org/10.4236/opj.2016.62004

[4] Marjani, S. (2013) Optimization of an InGaAsP Vertical-Cavity Surface-Emitting Diode Lasers for High-Power SingleMode Operation in $1550 \mathrm{~nm}$ Optical-Fibre Communication Systems. Asian Journal of Chemistry, 25, 4150-4152. http://dx.doi.org/10.14233/ajchem.2013.13186

[5] Faez, R., Marjani, A. and Marjani, S. (2011) Design and Simulation of a High Power Single Mode 1550nm InGaAsP VCSELs. IEICE Electronics Express, 8, 1096-1101. http://dx.doi.org/10.1587/elex.8.1096

[6] Marjani, S., Faez, R. and Hosseini, S.E. (2013) Threshold Characteristics Analysis of InP-Based PhC VCSEL with Buried Tunnel Junction. The 21st Iranian Conference on Electrical Engineering (ICEE), Ferdowsi University of Mashhad, Mashhad, 1-4. http://dx.doi.org/10.1109/iraniancee.2013.6599783

[7] Majdabadi, A., Marjani, S. and Sabaghi, M. (2014) Threshold Characteristics Enhancement of a Single Mode 1.55 $\mu \mathrm{mInGaAsP}$ Photonic Crystal VCSEL for Optical Communication Systems. Optics and Photonics Journal, 4, $296-303$. http://dx.doi.org/10.4236/opj.2014.410029

[8] Marjani, S., Faez, R. and Marjani, H. (2011) An Impact of the Hole Etching Depth within a Photonic Crystal VCSEL on Its Heat Sources. Australian Journal of Basic and Applied Sciences, 5, 766-770.

[9] Marjani, S., Faez, R. and Marjan, A. (2011) Design and Modeling of a High Single Mode Power Long Wavelength 
InGaAsP Photonic Crystal VCSEL. Australian Journal of Basic and Applied Sciences, 5, 1064-1069.

[10] Marjani, A., Marjani, S. and Shirazian, S. (2011) Numerical Simulation of Silicon Carbide Polymers (6H-SiC \& 3C-SiC) as the Active Area for $0.83 \mu \mathrm{m}$ Wavelength Semiconductor Laser. The 14th Iranian Physical Chemistry Conference, University of Tehran, Kish, 876-878.

[11] Marjani, S., Faez, R. and Marjani, H. (2011) Analysis and Design of Semiconductor Laser with Silicon Carbide Polymers (6H-SiC and 3C-SiC). Australian Journal of Basic and Applied Sciences, 5, 1060-1063.

[12] Marjani, S., Rahnama, M. and Marjani, H. (2011) Numerical Optimization of Single-Mode InGaAsP Vertical-Cavity Surface-Emitting Lasers. Australian Journal of Basic and Applied Sciences, 5, 1207-1211.

[13] Marjani, S. and Marjani, H. (2011) Effects of Lattice Temperature on the Various Elements of Heat Sources in a Long Wavelength InGaAsP Photonic Crystal VCSEL. Australian Journal of Basic and Applied Sciences, 5, 1257-1261.

[14] Marjani, S. and Marjani, H. (2011) Analysis of Lattice Temperature Effects on a Long Wavelength InGaAsP Photonic Crystal VCSEL. Australian Journal of Basic and Applied Sciences, 5, 1374-1378.

[15] Marjani, S. and Marjani, H. (2012) Optimization of a Long Wavelength Vertical-Cavity Surface-Emitting Lasers by Employing Photonic Crystal. Asian Journal of Chemistry, 24, 3174-3176.

[16] Marjani, S. and Marjani, H. (2012) Self-Heating Effects in a Silicon Carbide Polymers (6H-SiC and 3C-SiC) Semiconductor Laser. Asian Journal of Chemistry, 24, 3145-3147.

[17] Marjani, S. and Marjani, H. (2012) Effects of Hole Etching Depth in a Long Wavelength InGaAsP Photonic Crystal Vertical Cavity Surface Emitting Laser. Asian Journal of Chemistry, 24, 3194-3196.

[18] Marjani, S. and Marjani, H. (2012) Effects of Lattice Temperature on the Various Elements of Heat Sources in Silicon Carbide Polymers (6H-SiC and 3C-SiC) Semiconductor Laser. Asian Journal of Chemistry, 24, 3123-3125.

[19] Marjani, S., Faez, R. and Marjani, H. (2012) Analysis of the Various Elements of Heat Sources in Silicon Carbide Polymers (6H-SiC and 3C-SiC) Semiconductor Laser. Asian Journal of Chemistry, 24, 2333-2335.

[20] Marjani, S., Faez, R. and Marjani, H. (2012) Design and Modeling of a Semiconductor Laser by Employing Silicon Carbide Polymers (6H-SiC, 3C-SiC and 4H-SiC). Asian Journal of Chemistry, 24, 2177-2179.

[21] Marjani, S., Faez, R. and Hosseini, S.E. (2013) Analysis of Lattice Temperature Effects on a GaInP/6H-SiC Strained Quantum-Well Lasers. Asian Journal of Chemistry, 25, 4715-4717.

[22] Madadi, R., Marjani, S. and Faez, R. (2013) Silicon Carbide Polymers (6H-SiC, 3C-SiC and 4H-SiC) Semiconductor Laser: Influence of Self-Heating. The 3rd Iranian Conference on Optics and Laser Engineering (ICOLE), Isfahan, 8-10 October 2013, 1069-1072.

[23] Naeemi, M.A., Marjani, S. and Peiravi, A. (2014) Time to Failure Analysis of Single Mode Long-Wavelength InGaAsP Vertical-Cavity Surface-Emitting Lasers. The 22st Iranian Conference on Electrical Engineering (ICEE), Tehran, 20-22 May 2014, 43-47. http://dx.doi.org/10.1109/iraniancee.2014.6999500

[24] Khosroabadi, S., Keshmiri, S.H. and Marjani, S. (2014) Design of a High Efficiency CdS/CdTe Solar Cell with Optimized Step Doping, Film Thickness, and Carrier Lifetime of the Absorption Layer. Journal of the European Optical Society, 9, 14052-1-14052-6. http://dx.doi.org/10.2971/jeos.2014.14052

[25] Sabaghi, M., Majdabadi, A., Marjani, S. and Khosroabadi, S. (2015) Optimization of High-Efficiency CdS/CdTe Thin Film Solar Cell Using Step Doping Grading and Thickness of the Absorption Layer. Oriental Journal of Chemistry, 31, 891-896. http://dx.doi.org/10.13005/ojc/310232

[26] Sabaghi, M., Majdabadi, A., Khosroabadi, S. and Marjani, S. (2015) A Novel Ultrathin CdS/CdTe Solar Cell with Conversion Efficiency of $31.2 \%$ for Nano-Area Application. Proceedings of the Progress in Electromagnetics Research Symposium (PIERS), Prague, 6-9 July 2015, 1152-1155.

[27] Marjani, S., Khosroabadi, S. and Sabaghi, M. (2016) A High Efficiency Ultrathin CdTe Solar Cell for Nano-Area Applications. Optics and Photonics Journal, 6, 15-23. http://dx.doi.org/10.4236/opj.2016.62003

[28] Sabaghi, M., Majdabadi, A. and Oskui, H.S. (2007) Intelligent Controlling on Diode Laser Wavelength Based Embedded System. The International Universities Power Engineering Conference (UPEC), Brighton, 4-6 September 2007, 387-391.

[29] Withford, M.J., Brown, D.J.W., Mildren, R.P., Carman, R.J., Marshall, G.D. and Piper, J.A. (2004) Advances in Copper Laser Technology: Kinetic Enhancement. Progress in Quantum Electronics, 28, 165-196. http://dx.doi.org/10.1016/j.pquantelec.2003.12.001

[30] Little, C.E. (1999) Metal Vapor Lasers. John Wiley \& Sons, Chichester.

[31] Huang, Z.G., Namba, K. and Shimizu, F. (1986) Influence of Molecular Gases on the Output Characteristics of a Copper Vapor Laser. Japanese Journal of Applied Physics, 25, 1677-1679. http://dx.doi.org/10.1143/JJAP.25.1677

[32] Grant, B. (1997) Lasers Improve Uranium Enrichment. Photonics Spectra. http://www.photonics.com/Article.aspx?AID=1201 
[33] Behrouzinia, S., Sadighi, R. and Parvin, P. (2003) Pressure Dependence of Small-Signal Gain and Saturation Intensity of a Copper Vapor Laser. Applied Optics, 42, 1013-1018. http://dx.doi.org/10.1364/AO.42.001013

[34] Behrouzinia, S., Sadighi, R. and Parvin, P. (2004) Temperature Dependence of the Amplifying Parameters of a Copper Vapor Laser. Laser Physics, 14, 1050-1053.

[35] Lesnoi, M.A. (1984) Influence of Te Gas Mixture Composition and Cathode Material on the Output Power of Copper Vapor Laser. Soviet Journal of Quantum Electronics, 14, 142-148. http://dx.doi.org/10.1070/QE1984v014n01ABEH004664

[36] Ferrar, C.M. (1974) Buffer Gas Effects in a Rapidly Pulsed Copper Vapor Laser. IEEE Journal of Quantum Electronics, 10, 655-657. http://dx.doi.org/10.1109/JQE.1974.1068371

[37] Bokhan, P.A. and Solomonov, V.I. (1974) Mechanism of Laser Action in Copper Vapor. Soviet Journal of Quantum Electronics, 3, 481-486. http://dx.doi.org/10.1070/QE1974v003n06ABEH005640

[38] Withford, M.J., Brown, D.J.W., Coutts, D.W. and Piper, J.A. (1995) Copper Vapor Laser Unstable Resonator Oscillator and Oscillator-Amplifier Characteristics. IEEE Journal of Quantum Electronics, 31, 898-902. http://dx.doi.org/10.1109/3.375935

[39] Hayashi, K., Iseki, Y., Szuki, S., Watanabe, I., Noda, E. and Morimiya, O. (1992) Improvement in the Output Characteristics of a Large-Bore Copper Vapor Laser by Hydrogen. Japanese Journal of Applied Physics, 31, 689-697. http://dx.doi.org/10.1143/JJAP.31.L1689

[40] Khorasani, K., Behrouzinia, S. and Salehinia, D. (2008) Air as a Buffer Gas in Metal-Vapor Lasers. Journal of Russian Laser Research, 29, 599-603. http://dx.doi.org/10.1007/s10946-008-9047-9

[41] Zoghi, M., Parvin, P., Behrouzinia, S., Salehinia, D., Kharasani, K. and Mehravaran, H. (2009) Acoustic Effects of Metal-Vapor Lasers. Applied Optics, 48, 3460-3467. http://dx.doi.org/10.1364/AO.48.003460

[42] Kharasani, K., Salehinia, D., Behrouzinia, S., Sajad, B. and Parvizian, M. (2008) Frequency Dependence of the Output Power of Metal Vapor Lasers. Optics Communications, 281, 3799-3801. http://dx.doi.org/10.1016/j.optcom.2008.03.059 